\title{
TPM implantation in manufacturing systems: $A$ survey of the state-of-the-art and extensions
}

\author{
Kenza Chaabane*, Jérémie Schutz, and Sofiène Dellagi \\ Université de Lorraine, LGIPM, EA 3096, Metz, F-57045, France
}

\begin{abstract}
Maintenance activities represent a significant pillar in industry. Its real goal consists at improving the production systems productivity. Specially, TPM represents one of the main evolutions of the maintenance methods in order to improve the machines efficiency by a proactive approach. This paper presents an overview of several TPM implantations and especially efficiency and effectiveness measurement of the TPM in several industries. In a second hand, a proposition of possible extension about analytical assessment of the TPM performance, based on both technical and economic criteria, is presented.
\end{abstract}

\section{Introduction}

Companies are distinguished in terms of their industrial performance by the availability, productivity and flexibility of their resources as well as by ensuring lower expenses costs [1]. In today's dynamic environment, improving industrial performances is crucial for competitiveness, thus, companies should remove the unexpected (breakdowns, defects, waste of ...). In this context, maintenance has a very strategically role to play.

We note that several researchers have explored ways of integrating maintenance and production after decades during which those two activities had been studied separately. Works on maintenance policies started with Barlow et al. [2] and continued with a vast number of contributions as it can be seen in a survey on maintenance models by Wang [3]. During the last two periods, numerous companies have applied that strategies dissociating maintenance and production were unsuccessful. The real need for developing integrated maintenance-production strategies became compulsory. Brandolese et al. [4] proposed a strategy for maintaining a multiple machines production system. His planning consists in programming the execution date of each task and the machine that should perform it. They integrated preventive maintenance activities in the planning placing them as close as possible to the optimal maintenance periods. In the same framework, some researchers have taken into ac- count several external constraints. In fact, integrated maintenanceproduction strategies which take into consideration subcontracting have been studied by Dellagi et al. [5]

\footnotetext{
*Corresponding author: kenza.chaabane9@etu.univ- lorraine.fr, jeremie.schutz@univ-lorraine.fr, sofiene.dellagi@univ-lorraine.fr
} 
The TPM approach proposed in this survey deals with the frame of integrated production maintenance. In fact, works on TPM started in 1970's with Nakajima [6], he developed the concept of TPM when he improved the American method PM (Productive Maintenance) in Japanese company (Nippon Denso); since that, TPM Has refined over time, evolved according to the different experiments in different companies around the world. The purpose of this article is to present "the state of the art" in definition and implementation of TPM in manufacturing plant, and suggest a possible extension for measuring the assessment of the method which is rarely treated in literature. The reminder of this paper is organized as follows, the first section will review briefly some history, the target and expected results of the method. A second section will present some recent cases of study of the implementation of TPM in different companies. section 3 is dedicated to the comparison of the different ways to assess TPM improvement and propose a possible extensions based on both technical and economic criteria. Finally, conclusions and potential future work are provided in Section 4.

\section{Presentation of the TPM method}

The original TPM was mediated by Seichi Nakajima, Director of JIPM (Japan Institute of Plan Maintenance). His concept was "Creating small groups to take ownership of equipment and improve its performance" [6]. This concept stems from big changes in the company, namely that we are no longer in the basic scheme "I produce, you repair ", but in a scheme where maintenance is everyone's busi- ness. This is reflected in the fact that the operator performs some of the so-called "repair level" such as lubrication, monitoring, cleaning and control; in the 70'th, this vision was innovative and encountered a lot of opposition but since then, success has swept away all fears.

Nakajima [6] define TPM as "productive maintenance involving total participation in addition to maximizing equipment effectiveness and establishing a through system of planned maintenance. Also, the JIPM defined TPM as a system of Maintenance covering the entire life of the equipment including manufacturing, planning and maintenance. The world "total" in TPM has three principal meanings which are the feather of the TPM: the first means total effectiveness indicates TPM's pursuit of economic efficiency, the second is for total maintenance system including maintenance prevention, maintainability improvement and preventive maintenance. The last meaning and the most important is for total participation of all employees, from the top management to workers on the shop floor.

Thus, TPM is closely tied to "just in time", $5 \mathrm{~S}$ and "total quality management"; also, it is an extension of "PM", where the machine works at high productivity and efficiency, and where the maintenance is all employee's responsibility, focusing to prevent the problems before it may occur. Referring to bufferne [1], the principal goals of the TPM are: Improve equipment effectiveness by looking into the six big losses, to make the equipment work at it highest performance; reduce production's direct and indirect costs; enhance the life cycle of equipment and reduce it cost by improving maintenance efficiency and effectiveness and create an operational value for the company by educating and training personnel witch is an important value added of the TPM approach.

Notice that despite the efforts made, some companies fail to deploy TPM within their production systems. The reasons are numerous and constitute barriers to the implementation of this proactive strategy. In his study, Kumar et al. [7] identified various causes and proposed a model to analyze and eliminate them later. One of the most important causes is the lack of involvement of the manager and human resources. We must also cite the resistance to change of employees who sometimes have trouble getting out of the traditional pattern (I produce, maintenance repairs). The lack of communication 
between the departments also constitutes a barrier to the implementation of the method, hence the need to set up pyramidal structures.

\section{Effectiveness and efficiency measurement: cases of study}

\subsection{Effectiveness measurement}

Industrials affirm that the performance measurement system is a key element in order to motivate the whole staff (We progress only if we measure a performance). In the TPM approach, the OEE (Overall Equipment Effectiveness) is the basic indicator to which other indicators can be added. Since the aim of the TPM is to achieve ideal performance and zero losses, quantifying the accumulation of time waste is the best way to measure the equipment effectiveness, then by definition:

$$
\mathrm{OEE}=\text { Valuable operating time / Loading time [1] }
$$

Where loading time is the total time available to produce in a given period and the valuable operating time is only the time use to fabric good products.

It is also expressed by the relation [1]:

$$
\mathrm{OEE}=\text { Availability } \times \text { Performance rate } \times \text { Quality rate }[1]
$$

The availability is calculated as:

$$
\text { Availability }=(\text { Required availability }- \text { Downtime }) / \text { Required availability }[1]
$$

The formula to compute the performance rate can be expressed as:

$$
\text { Performance rate }(\mathrm{P})=(\text { Design cycle time } x \text { Output }) / \text { Operating time [1] }
$$

The design cycle time or the production output will be in the unit of production, like parts per hour and the output will be the total output in the given time period interval. The quality rate can be expressed as:

$$
\text { Quality rate }(\mathrm{Q})=(\text { Production input-Quality defects }) / \text { Production input [1] }
$$

The production input means that the unit of product being feeds into the production process. The quality defects mean the number of products which are below the quality standards i.e. the rejected items after the production process.

In 2016, Kumar et al. [7] studies the relation between OEE, A, P and Q. By looking at the values of correlation, he came to conclusion that OEE is highly correlated with Availability then Performance rate and then Quality rate. The paper also compares the effectiveness of the TPM implementation between two plastic industries in India using the OEE; it shows that both industries are lagging in effective implementation of important pillars of the TPM when comparing there OEE to the world standard OEE; Still Company A has got a little bit better implementation of TPM than Company B, same time OEE value of Company $\mathrm{A}$ is higher than Company B by $12 \%$.

Singh et al. [8] study the implementation of the TPM in a Machine Shop in Indian automotive company. The paper shows detailed steps of the implementation of the method, it also compares OEE before and after TPM implementation, it has improved from $63 \%$ to $79 \%$ indicating the improvement in productivity and improvement in quality of products. 
In 2016, Vittaleshwar et al. [9] establish an empirical study of effect of Total Productive Maintenance on OEE in a Water Bottling Industry. Data was collected on daily activities and was used for calculation of OEE for Water Treatment Plant, Bottle Blowing Unit, and Bottle Filling Machine 30

Bottles per Minute, Pouch Packing Machine and Inkjet Jet Coders before and after TPM implementation. The average value of OEE were found fall between the ranges of 0.14 and 0.7. The world-class benchmarks for OEE is 0.85. TPM and OEE helped the Water Bottling Company to identify previously hidden manufacturing losses and inefficiency.

We can deduct from various cases of study seen in this part, that the OEE is not a simple indicator. it is a tool of progress which allows the identification of possible hidden losses, to better eliminate them.

\subsection{Efficiency measurement: DEA method}

When it comes to performance evaluation in TPM, OEE has commonly been used as a performance measure since TPM aims to maximize equipment effectiveness. Although OEE has been considered as a standard measure for equipment performance what it captures is only effectiveness of TPM, not efficiency. However, the performance evaluation of TPM should include an objective and a method based on multiple different factors (inputs and outputs) [10]. The DEA method is a linear program- ming model for evaluating the relative efficiency of comparable decision-making units (DMUs) in transforming inputs into outputs in a direct fashion without recourse to any underlying model [11]. It is a non-parametric approach that does not require any assumptions about the functional form of a production function or a priori information on importance of inputs and outputs [12]. There are two models of the DEA, the first (CCR model for Charnes, Cooper, and Rhodes, 1978) assumes that production exhibits constant returns to scale; that is, if an activity ( $\mathrm{x}, \mathrm{y})$ is feasible under a given production frontier, then, for every positive scalar $\mathrm{t}$, the activity (tx, ty) is also feasible.

The second model (BCC for Banker, Charnes, and Cooper (1984)) for encompassing variable returns to scale along the efficiency frontier. DEA models are distinguished by the objective of a model: maximize outputs (output-oriented) or minimize inputs (inputoriented). Since it is implicitly assumed that the objective of TPM lies in increasing outputs rather than decreasing inputs, also it cannot be assumed that TPM activities exhibit constant returns to scale [13]. More precisely, it is the output-oriented BCC model which is used to measure TPM efficiency, it is formulated as the following [14]:

$$
\begin{aligned}
& \min \theta_{\mathrm{p}} \\
& \text { st } \sum_{\mathrm{j}=1}^{\mathrm{n}} \lambda_{\mathrm{j}} \cdot \mathrm{x}_{\mathrm{ij}} \leq \theta_{\mathrm{p}} \cdot \mathrm{x}_{\mathrm{ip}} \quad \forall \mathrm{i}, \\
& \sum_{\mathrm{j}=1}^{\mathrm{n}} \lambda_{\mathrm{j}} \cdot \mathrm{y}_{\mathrm{rj}} \geq \mathrm{y}_{\mathrm{rp}} \quad \forall \mathrm{r}, \\
& \sum_{\mathrm{j}=1}^{\mathrm{n}} \lambda_{\mathrm{j}}=1, \quad \lambda_{\mathrm{j}} \geq 0, \forall \mathrm{j} .
\end{aligned}
$$

Jeon et al.[13] study measured the efficiency of TPM using DEA where SDWT was the DMUs using DEA. It considered that TPM implementation process is captured in a three-stage model: stage 1 from TPM inputs (employee, expense) to TPM intermediate outputs (autonomous maintenance, planned maintenance, education and training), stage 2 
from TPM intermediate outputs to TPM final outputs (availability, performance efficiency), and stage 3 from TPM inputs to TPM final outputs. Every SDWT in every team was evaluated by DEA for each stage. Based on the efficiency scores of DEA, the relationships between stage-wise efficiency scores of SDWTs is analyzed by correlation analysis. It finds that SDWTs efficient transforming intermediate outputs into final outputs are also efficient at transforming inputs into final outputs.

Bekar et al. [10] go further to deal with situations where some of the input and output data for TPM are imprecise or not available, they used a newly method where fuzzy logic is introduced to DEA for measuring efficiency of DMUs under uncertainty. It's called Fuzzy data envelopment analysis (FDEA).

In the study, the performance efficiency of TPM for four production lines (DMUs) was evaluated (Rail Machining, Rail Assembly and HPV, NHB, Beginning of Line and NHB End of Line) with respect to seven outputs and ten inputs, the fuzzy weights of inputs and outputs are obtained by fuzzy COPRAS. After estimation, the study shows that the rail machine was on efficient frontiers, it is $100 \%$ efficient, unlike rail assembly and HPV which was the lowest efficient. This study is the first introducing FUZZY COPRA-FUZZY DEA method to evaluate performance efficiency of TPM.

\section{Possible extensions}

Looking at the literature related to TPM, detailed in last section, we have seen different methods to evaluate TPM implementation in companies, depending on what we want to improve (efficiency or effectiveness). In the other hand, those methods still too relatives results.

For example, the JIPM precise that it's not the number itself that count for OEE, but how much we improve it, it depends on the ideal objective that we fixed and how much did we approach it, that's the meaning of the effectiveness measuring. For example, Buffern [1] says that in a Japanese company he visited, the OEE presented was $110 \%$, it's explained by the fact that progress realized by the company during the 4 years of TPM implementation exceeds the initial too pessimist objectives. It proves that OEE still a relative factor.

The DAE method, as for her, aims to compare different units (DMUs) in same company relative to their best peers (efficient frontier). DMUs which belong to this frontier are $100 \%$ efficient, even if the TPM wasn't well implemented in the company.

Also, the OEE measurement, which is a function of availability, performance and quality rate need large amount of data collection. As Hammar et al. [15] says that "The data collection should be at such a detailed level that it fulfils its objectives without being unnecessarily demanding resources. Too detailed data collection may result in unmotivated personnel and reaction against the measurement". Moreover, it's mentioned that "To succeed with data collection, it is necessary to find a less time consuming method that is also precise". These statements show that a simple measure of OEE and co-operation of production personnel are the crucial factors for successful data collection.

A new measuring method or economical or technical estimation of the efficiency of the TPM is compulsory at this stage using less data measurement, and giving non relative assessment. Looking to the evolution in studies related to preventive maintenance and new integrated maintenance production strategies. These studies started by simple description or proposition of maintenance strategies [3] in order to reach recently a hay level research evolving a real estimation of economical or technical strategies. More then, in several studies the authors developed an analytical model representing an objective function related to economical or technical profit. The authors optimize analytically or by simulation methods theses developed functions in order to obtain an optimal variable decisions helpful for industrial (Schutz et al. [16], Hajej et al. [17]). 
Dealing with this context, our idea consists at introducing a new variable which related to the time spent by operator on doing first level maintenance. In fact, one of the most important added value of the TPM is first level maintenance. We find the optimal time spending by that operator on checking the machine in its work-time, noted TPM period, ensuring compromise between system reliability and economical profit related to selling costs, production costs and others relative dispenses.

The benefice related to maintenance after TPM implementation will be the new measurement of its efficiency and depends on this new variable, it could be estimated analytically or by simulation, according to the complexity of the developed model, in the aim to maximize it as an objective function.

\section{Conclusion}

The purpose of this report was to present a state-of-the-art of the TPM approach, in order to better identify the method. We first considerate the origin of the approach and its objectives. Then, we focused at measuring the effectiveness and efficiency of TPM; the various indicators that we used, the OEE for efficiency and the DEA method for performance measurement, were presented based on the latest studies on TPM over the last decade.

After reflection, it was possible to say that the indicators that were used were limited; also, in the literature, rare are the documents which are interested in measuring the effectiveness of the TPM using economic criteria.

Following in this study, it is proposed to develop an economical way to evaluate the performance of the TPM, which will have as objective function the gain maximization.

\section{References}

1. J. Bufferne, Le guide de la TPM: Total Productive Maintenance (Editions Eyrolles, 2006), ISBN 978-2-7081-3723-3, google-Books-ID: F5A8y88nn7cC

2. R.E. Barlow, F. Proschan, Studies in Applied Probability and Management Science 7, 63 (1962)

3. H. Wang, European Journal of Operational Research 139, 469 (2002)

4. M. Brandolese, M. Franci, A. Pozzetti, International Journal of Production Research 34, 2059 (1996)

5. S. Dellagi, N. Rezg, X. Xie, International Journal of Production Research 45, 1233 (2007)

6. S. Nakajima, Introduction to TPM: Total Productive Maintenance (Productivity Press, 1988), ISBN 978-0-915299-23-2, google-Books-ID: XKc28H3JeUUC

7. A. Kumar, R.K. Singh, T.A. Khan, International Journal of Advance Research and Innovation 3, 82 (2016)

8. R. Singh, A.M. Gohil, D.B. Shah, S. Desai, Procedia Engineering 51, 592 (2013)

9. A. Vittaleshwar, K.S. Dasharathraj, P. Prajual, International Journal of Applied Engineering Research 11, 5573 (2016)

10. E.T. Bekar, C. Kahraman, Measuring Efficiency of Total Productive Maintenance (TPM) with Newly Developed Performance Measures using Fuzzy Data Envelopment Analysis, in Proceed- ings of the World Congress on Engineering (2016), Vol. 1

11. W.W. Cooper, L.M. Seiford, J. Zhu, Socio-Economic Planning Sciences 34, 1 (2000)

12. W.D. Cook, L.M. Seiford, European journal of operational research 192, 1 (2009) 
13. J. Jeon, C. Kim, H. Lee, Total Quality Management \& Business Excellence 22, 911 (2011)

14. A. Charnes, W.W. Cooper, Accounting, Organizations and Society 5, 87 (1980)

15. P. Hammar, B. Bennett, M. Yang, M. Johnson, Physical Review Letters 83, 203 (1999)

16. J. Schutz, Joint optimization of production-maintenance plans based on optimal production rates, in 2014 IEEE International Conference on Industrial Engineering and Engineering Management (2014), pp. 933-937

17. H. Zied, D. Sofiene, R. Nidhal, International Journal of Production Research 49, 5695 (2011) 